\title{
Stellar Object Detection Using the Wavelet Transform
}

\author{
E. Anisimova, P. Páta, M. Blažek
}

\begin{abstract}
Several algorithms are used nowadays for detecting stellar objects in astronomical images, for example in the DAOPHOT program package and in SExtractor (Software for source extraction). Our team has become acquainted with the wavelet transform and its good localization properties. After studying the manual for DAOPHOT and SExtractor, and becoming familiar with the à trous algorithm used for calculating the wavelet transform, we set ourselves the task to implement an algorithm for star detection on the basis of the wavelet transform. We focused on detecting stellar objects in complex fields, such as globular clusters and galaxies. This paper describes a stellar object detection algorithm with the help of the wavelet transform, and presents our results.
\end{abstract}

Keywords: stellar object detection, wavelet transform.

\section{Introduction}

The DAOPHOT program [1,2] and SExtractor $[3,4]$ calculate the estimated background value and perform thresholding of each pixel: if it is more than a specified threshold and meets certain conditions, we consider it to be a light source. Otherwise we assume that it is noise. Problems arise in the case of faint stars, whose brightness is close to the ambient background. For this reason, they may not be properly detected. Multiresolution methods of image analysis, e.g. the wavelet transform, have therefore gained ground. The main advantage of this transform is its ability to separate light sources contained in an image according to their size, enabling us to analyze both large, bright objects and the small, faint stars in their neighborhood.

\section{Wavelet transform of a 2D image}

To realize the wavelet transform of an image it is necessary to make an image convolution with a preselected wavelet, first by rows and then by columns. The result is an approximation of the original image and the presence of details in horizontal, vertical and diagonal direction. With increasing decomposition level we extract larger details from the image. In order not to change the scale of the low-pass or highpass wavelet filters, the image has to be subsampled by a factor of 2 . Therefore, when the wavelet transform is implemented by the Mallat algorithm [5] there is for each additional degree of decomposition an image with dimensions twice smaller than on the previous level. For detecting stellar objects, this is not a good property, because we need to have the same number of pixels on each scale in order to comply with the same coordinates. Therefore, the wavelet transform for astronomical purposes is realized by the à trous algorithm ("with holes").

\section{The à trous algorithm}

Wavelet transform implementation by the à trous algorithm involves convoluting the input image with a $2 \mathrm{D}$ convolution kernel representing a two-dimensional scaling function, which imitates the stellar PSF [6].

To imitate the subsampling process, we have to change for each next decomposition level the filter length in such a way that $2^{j}-1$ zeros are inserted between the coefficients, where $j$ is the decomposition level [6].

- During the first decomposition (we start from $j=0$ ) we convolute the original image $S_{0}$ with an unmodified kernel $K_{0}$, and the result is the smoothed matrix $S_{1}$.

- Subtracting $S_{1}$ of $S_{0}$, we get wavelet coefficients for the first decomposition level corresponding to the smallest details $W_{1}=S_{0}-S_{1}$.

- $j=j+1$.

- Expand the filter by $2^{j}-1$ zeros.

- Calculate the smoothed matrix $S_{2}=S_{1} \star K_{1}$ and the wavelet coefficients for the second decomposition level: $W_{2}=S_{1}-S_{2}$, etc. If we stop this algorithm here, the original image is the sum of $S_{2}, W_{1}$ and $W_{2}$ (Figure 1).

\section{Detecting stellar objects}

Stellar objects are detected in wavelet coefficients $W_{1}, W_{2}$, etc., representing details contained in the original image. This means that stars with the nar- 


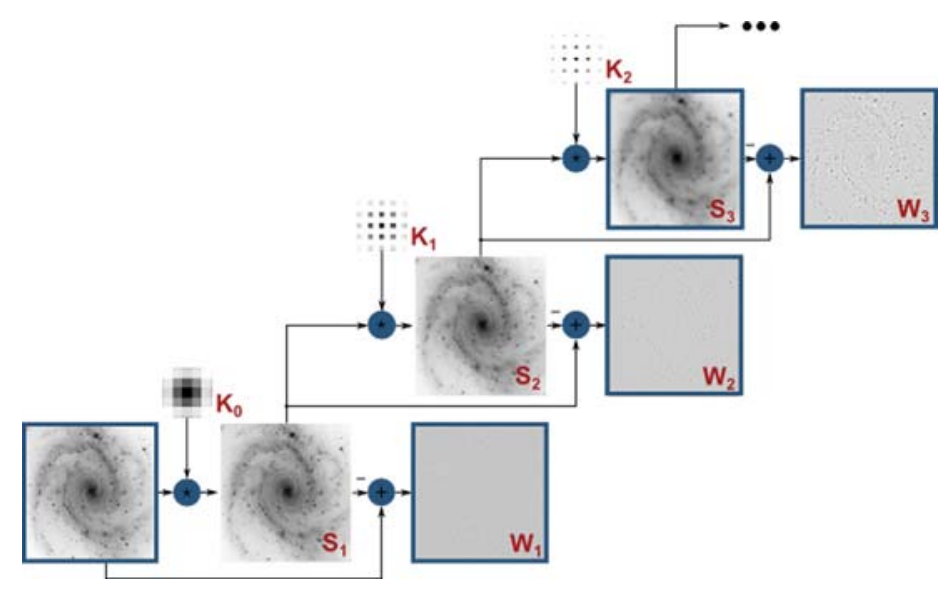

Fig. 1: The à trous algorithm [8]

rowest radial profile are detected in $W_{1}$ and with increasing decomposition level flatter and more extensive objects will be found.

1. After calculating the à trous decomposition of the image we determine their significance at each level of wavelet coefficients. This is done by noise level, or by estimating the level of the stellar background (we start as in the case of conventional algorithms). A robust estimate $\hat{\sigma}$ is obtained with a median measurement, which is highly insensitive to isolated outliers of potentially high amplitudes [7]. This estimate is usually used to remove noise from the image, but we will use it to determine the threshold and to distinguish between significant coefficients belonging to stellar objects and insignificant coefficients that are part of the background.

2. The estimated noise standard deviation for each decomposition level will be used for wavelet coefficient thresholding in a way that it will set to zero all the coefficients belonging to the interval $\left|W_{J}\right| \leq 3 \hat{\sigma}$.

3. Among the non-zero coefficients we then look for the local maxima of the wavelet coefficients.

4. The coordinates of the local maxima can be considered to be stellar detections if there are nonzero wavelet coefficients in the next decomposition level in the same places. In this way we verify whether the detected objects have a star shape, i.e. we try to eliminate the detection of hot pixels and the detection of false centers, which could be detected due to imperfect background level estimation.

\section{Results}

In this paper the proposed algorithm for stellar object detection is based on the wavelet transform. Our team also carried out stellar detection by standard algorithms (used in SExtractor, with subtraction of discovered stars [8]) as well as by the wavelet transform. We monitored the total number of detected stars, the time required for performing detection, the number of discovered stars corresponding to optical catalog USNO-A2.0 [9], and the percentage of the total number of stars in the catalog for a given image. The best results (number of real detected stars, and the time spent) were achieved using an algorithm based on the wavelet transform.

Individual images were taken by the D50 Telescope at the Astronomical Institute of the Academy of Sciences of the Czech Republic at Ondrejov by the High Energy Astrophysics Group. Table 1 illustrates the results for number of detected light sources for the following stellar objects and their neighbourhood: GRB 100902A (Gamma-Ray Burst), M5 (Globular Cluster), M67 (Open Cluster) and M51 (Galaxy). Table 2 shows the results compared with the USNOA2.0 catalog. For each image there are in the first line: the number of detected stars with the coordinates found in the catalog, the number of detected stars not found in the catalog, the percentage of detected stars with coordinates found in the catalog out of the total number of objects in the catalog for the image (second row).

Figure 2 compares the number of detected light sources using conventional methods based on background estimation and also using the wavelet transform.

Table 1: Number of detected light sources.

\begin{tabular}{|c|c|c|}
\hline Image & $\begin{array}{c}\text { Standard } \\
\text { algorithm }\end{array}$ & $\begin{array}{c}\text { Wavelet } \\
\text { transform }\end{array}$ \\
\hline GRB 100902A & 402 & 513 \\
\hline M 5 & 1031 & 1918 \\
\hline M 67 & 368 & 499 \\
\hline M 51 & 141 & 1191 \\
\hline
\end{tabular}


Table 2: Number of discovered stars corresponding to optical catalog USNO-A2.0 and the percentage of the total number of stars in the catalog for a given image

\begin{tabular}{|c|c|c|c|c|c|c|}
\hline \multirow{2}{*}{ Image } & \multicolumn{3}{|c|}{ Standard algorithm } & \multicolumn{3}{c|}{ Wavelet transform } \\
\cline { 2 - 7 } & In catalog & Outside catalog & {$[\%]$} & In catalog & Outside catalog & {$[\%]$} \\
\hline GRB 100902A & 367 & 35 & 62 & 401 & 112 & 68 \\
Total & 592 & & & 592 & & \\
\hline M 5 & 823 & 208 & 16 & 1023 & 895 & 20 \\
Total & 5213 & & & 5213 & & \\
\hline M 67 & 355 & 13 & 38,5 & 445 & 54 & 48 \\
Total & 922 & & & 922 & & \\
\hline M 51 & 63 & 78 & 68 & 88 & 1103 & 95 \\
Total & 93 & & & 93 & & \\
\hline
\end{tabular}

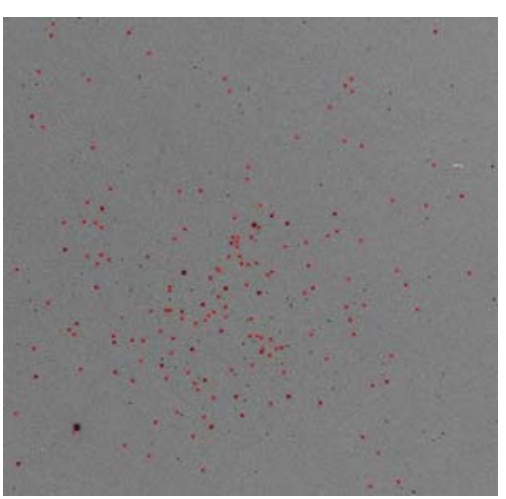

(a)

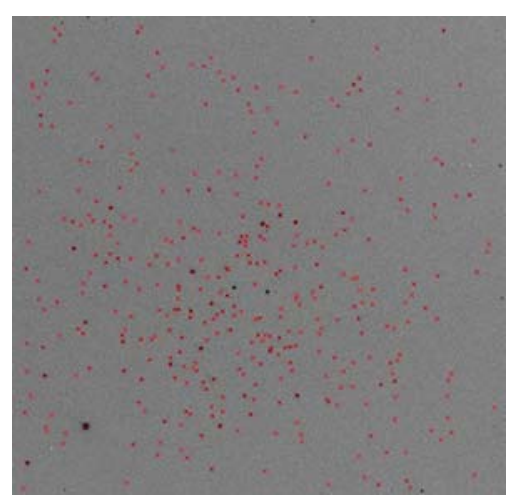

(b)

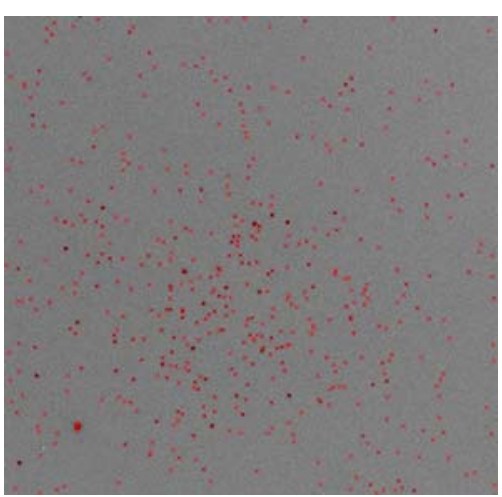

(c)

Fig. 2: An example of stellar object detection for an image of open cluster M67: using conventional methods based on estimation of the global (a) and local (b) background and using the wavelet transform (c) [8]

\section{Conclusion}

The best detection results were achieved using a method based on image analysis using the wavelet transform: the total number of discovered objects and the percentage of the number of stars in the catalog for all images is the highest of all tested methods [8]. In addition, there is an interesting situation. In simpler stellar fields (GRB 100902A, M 67) we found more objects that are also listed in the catalog, while the situation was the opposite for a globular cluster and for galaxy M 51. This is due to the fact that stars in the middle of globular clusters or galaxies are not recorded in the catalog, so all light sources found in this place will be evaluated as not belonging to the catalog. Conversely, there are many stars in the catalog away from the middle of a cluster that are almost indistinguishable from noise, or not at all visible, so they have not been detected.

\section{Acknowledgement}

This paper has been supported by grant project SGS10/285/OHK3/3T/13.

\section{References}

[1] DAOPHOT - Stellar Photometry Package [online]. Web site of the software package. [cit. 2011-28-05]. Available on the Web: http://www.star.bris.ac.uk/ mbt/daophot/

[2] Stetson, P. B.: DAOPHOT: A computer program for crowded-field stellar photometry. $P u b$ lications of the Astronomical Society of the Pacific. March 1987, 99, p. 191-222.

[3] Bertin, E., Arnouts, S.: SExtractor: Software for source extraction. Astronomy and astrophysics supplement series. June 1996, 117, p. 393-404.

[4] SExtractor - Astronomical Source Extractor [online]. Web site of the software package. [cit. 2011-28-05]. Available on the Web: http://sextractor.sourceforge.net/

[5] Mallat, S. G.: A Theory for Multiresolution Signal Decomposition: The Wavelet Represen- 
tation. IEEE Transactions on Pattern Analysis and Machine Intelligence. July 1989, vol. 11, no. 7 .

[6] Starck, J.-L., Murtagh, F.: Astronomical Image and Data Analysis. Springer-Verlag Berlin Heidelberg, 2002. 289 p. ISBN 3-540-42885-2.

[7] Donoho, D. L., Johnstone, I. M.: Ideal Spatial Adaptation by Wavelet Shrinkage. Biometrika. September 1994, vol. 81, p. 425-455.

[8] Anisimova, E.: Methods for Analysing and Processing of Astronomical Image Data. Prague, 2011. 77 p. Thesis. Czech Technical University in Prague, Faculty of Electrical Engineering, Department of Radio Engineering.

[9] USNO - A2.0. A Catalog of Astrometric Standards [online]. [cit. 2011-05-05]. Available on the Web: http://tdc-www.harvard.edu/software/ catalogs/ua2.html

Elena Anisimova

Petr Páta

Martin Blažek

Department of Radio Engineering

Faculty of Electrical Engineering

Czech Technical University in Prague 